\title{
ASPIRAÇÃO ENDOTRAQUEAL: ESTUDO EM PACIENTES DE UMA UNIDADE DE URGÊNCIA E TERAPIA INTENSIVA DE UM HOSPITAL DA REGIAO METROPOLITANA DE NATAL - RN ${ }^{1}$
}

\author{
INTRATRACHEAL SUCTION: STUDY IN PATIENTS OF AN EMERGENCY AND INTENSIVE CARE UNIT IN A \\ HOSPITAL OF THE METROPOLITAN REGION OF NATAL CITY - RN
}

\begin{abstract}
ASPIRACIÓN DE LAS VIAS AÉREAS SUPERIORES: ANÁLISIS EM ENFERMOS DE UNA UNIDAD DE URGENCIA Y TERAPIA INTENSIVA DE UN HOSPITAL EN LA REGIÓN METROPOLITANA DE NATAL - RN
\end{abstract}

\section{Glaucea Maciel de Farias ${ }^{2}$, Izaura Luzia Silvério Freire ${ }^{3}$, Cristiane da Silva Ramos ${ }^{4}$}

RESUMO: A aspiração endotraqueal é um recurso mecânico simples e importante na rotina hospitalar. É amplamente utilizado em pacientes em Unidade de Terapia Intensiva (UTI), sob ventilação mecânica ou não. Objetivamos nesse estudo caracterizar os profissionais que realizam o procedimento de aspiração das vias aéreas superiores e identificar como está sendo realizado esse procedimento. A pesquisa é do tipo exploratório descritivo com dados prospectivos e abordagem quantitativa, realizada nas Unidades de Urgência e UTI de um Hospital da grande Natal-RN. A população constou de 14 enfermeiros, 22 técnicos de enfermagem, 14 auxiliares de enfermagem, 03 médicos e 07 fisioterapeutas. Empregou-se na coleta dos dados a técnica de observação estruturada. $O$ instrumento constou de um protocolo de aspiração baseado nas diretrizes da Agência Nacional de Vigilância Sanitária, Ministério da Saúde e o do Guidelines for Prevention of Nosocomial Pneumonia do Centro de Controle de Doenças (CDC, 1997). Constatamos que as diretrizes norteadoras deste procedimento não eram levadas em consideração em quase todos os passos deste cuidado. O que mais chamou a atenção foi a falta da lavagem das mãos antes (92,81\%) e após $(50,29 \%)$ este cuidado. A pouca participação do enfermeiro, $(3,59 \%)$ nesta atividade também é considerado um ponto de extrema relevância por se tratar de unidade de cuidados de alta complexidade. Os dados apontam a necessidade de intensificar as atividades educativas que promovam a mudança de comportamento destes profissionais, melhorando assim a qualidade da assistência e a prevenção de infecções nosocomiais.

PALAVRAS CHAVES: Cuidados Intensivos; Qualidade da Assistência à Saúde; Cuidados de Enfermagem.

ABSTRACT: Intratracheal suction is a simple and important mechanical procedure on hospital routine. It's largely used on patients in Intensive Care Units (ICU), under mechanical ventilation or not. This study seeks to characterize the professionals that perform the procedure of upper air tracts aspiration and identify how this procedure is being performed. The research is of the descriptive exploratory type with prospective data and quantitative approach, performed on the Emergency Room and ICU of a hospital in the greater Natal-RN. The population is comprised of 14 nurses, 22 nursing technicians, 14 nursing auxiliaries, 03 physicians and 07 physiotherapists. The structure observation technique was employed in the data collection. The instrument was comprised of a suction protocol based on the directives of the Agência Nacional de Vigilância Sanitária, Ministério da Saúde, and of the Guidelines for Prevention of Nosocomial Pneumonia of the Center for Disease Control (CDC, 1997). We've discovered that the guidelines for this procedure were not taken into consideration in almost every step of this care. The most outstanding discovery was the absence of hand washing before $(92,81 \%)$ and after $(50,29 \%)$ this care. The small participation of the nurse $(3,59 \%)$ on this activity is also considered a point of extreme relevance, since it's a unit for care of extreme complexity. The data indicate the need to intensify the educating activities that promote a change of behavior on these professionals, thus improving the quality of assistance and prevention of nosocomial infections.

KEYWORDS: Intensive Care; Quality of Health Care; Nursing Care.

RESUMEN: La aspiración endotraqueal es un recurso mecánico muy sencillo e importante en la rutina hospitalar. Es utilizado con mucha frecuencia en enfermos ingresados en unidad de terapia intensiva (UTI), bajo ventilación mecánica o no. Objetivamos en ese trabajo caracterizar los profesionales que realizan el procedimiento de aspiración de las vías aéreas superiores e identificar de que modo está siendo realizado ese procedimiento.

${ }^{1}$ Trabalho da Dissertação de Mestrado em Enfermagem do Curso de Pós-Graduação em Enfermagem da Universidade Federal do Rio Grande do Norte.

${ }^{2}$ Enfermeira. Doutora em Enfermagem. Professora da Graduação e Pós-graduação da Universidade Federal do Rio Grande do Norte (UFRN), Natal, RN, Brasil. E-mail: glaucea@digizap.com.br

${ }^{3}$ Enfermeira Intensivista, Mestra do Departamento de Enfermagem da Universidade Federal do Rio Grande do Norte (UFRN), Natal, $R N$, Brasil. E-mail: izaurafreire@hotmail.com

${ }^{4}$ Enfermeira. Mestranda em Enfermagem do Departamento de Enfermagem da Universidade Federal do Rio Grande do Norte (UFRN), Natal, RN, Brasil. E-mail: cristiane ramos@hotmail.com 
La pesquisa es del tipo exploratorio descriptivo con datos prospectivos y abordaje cuantitativo, realizada en las unidades de urgencia y UTI de un hospital alrededor de Natal. La población era formada por 14 enfermeros, 22 técnicos de enfermería, 14 auxiliares de enfermería, 03 médicos y 07 fisioterapeutas. Se ha empleado en la colecta e datos, la técnica de observación estructurada. El instrumento consistía de un protocolo de aspiración basado en las directrices de la agencia nacional de vigilancia sanitaria, Ministerio de la Salud y del Guidelines for Prevention of Nosocomial Pneumonia del Centro de Control de Enfermedades (CDC, 1997). Hemos constatado que las directrices orientadoras de ese procedimiento no eran llevadas en cuenta en casi todos los pasos lo

\section{INTRODUÇÃO}

A aspiração endotraqueal é um recurso mecânico simples e importante na rotina hospitalar. É amplamente utilizado em pacientes em Unidade de Terapia Intensiva, sob ventilação mecânica ou não, ou em pacientes de leito hospitalar que não conseguem expelir voluntariamente as secreções pulmonares traqueobrônquicas, sangue e vômitos, sem traumatismos, com a finalidade de manter as vias aéreas permeáveis, prevenir infecções, promover trocas gasosas, incrementar a oxigenação arterial e melhorar a função pulmonar (COSTA, 1999).

Este procedimento expõe a sérios riscos, especialmente em pacientes sob ventilação mecânica, devendo ser cuidadosa e criteriosa para evitar complicações sérias como hipoxemia, atelectasia, arritmia e infecção, entre outras (COSTA, 1999).

Para realizar a aspiração das vias aéreas, se faz necessário que se tenha à mão: estetoscópio, fonte de oxigênio e conexões, sistema de vácuo e conexões, monitor cardiorrespiratório e saturímetro, óculos protetores, máscara facial, avental descartável, luva estéril, sondas de aspiração traqueal adequada à idade ou a compleição física, solução fisiológica a 0,9\%, compressa estéril, gaze estéril, seringas e ambu (HINRICHSEN, 2004).

$\mathrm{Na}$ aspiração devem ser usadas sondas traqueais maleáveis, descartáveis, nos tamanhos 12 ou 14, com três orifícios (no mínimo) na extremidade distal, dispostos lateralmente e na ponta, para que não haja colabamento da traquéia, que poderia provocar ulcerações e sangramentos. É recomendável que possua válvula digital para controle da pressão do vácuo e que o diâmetro externo não exceda $1 / 3$ do diâmetro interno do tubo endotraqueal ou traqueóstomo. Durante a aspiração pode ser necessário o suporte de oxigênio de acordo com o quadro clínico do paciente (COSTA, 1999).

Para que seja realizada de forma asséptica, devemos antes de iniciar o procedimento lavar as mãos com técnica correta, abrir a ponta do papel da que más llamó la atención ha sido la falta de limpieza de las manos antes $(92,81 \%)$ y después $(50,29 \%)$. La participación muy pequeña de los enfermeros $(3,59 \%)$ en esta actividad también es considerada un asunto de extrema importancia por tratarse de unidad de cuidados de alta complicación. Los datos apuntan hacia la necesidad de intensificar las actividades educativas que promuevan la mudanza de comportamiento de eses profesionales mejorando de esa manera la calidad de la asistencia y la prevención de infecciones nosocomiales.

PALABRAS CLAVES: Cuidados Intensivos; Calidad de la Asistencia; Atención de Enfermería.

sonda estéril, adaptá-la à conexão do vácuo, abrir o vácuo e, em seguida, calçar luvas estéreis, segurando-a com uma das mãos e com a outra desconectando o respirador. Em seguida, introduz-se o cateter na traquéia do paciente através do tubo endotraqueal ou traqueostomia o qual deverá estar ligado a um sistema aspirador; a aspiração será realizada quando a ponta do cateter estiver no interior da traquéia (DREYER et al, 2003).

O tempo da introdução da sonda deve ser o mais rápido possível, e sua retirada deve ser feita com movimentos circulares, produzidos com os polegares e indicador, permitindo a limpeza das secreções com o mínimo de dano à parede traqueal. A duração não deve ser superior a 10 segundos, pois o fator tempo é um determinante muito importante, uma vez que o conteúdo aéreo nos pulmões fica reduzido, podendo levar a hipóxia, já que, juntamente com as secreções, aspira-se ar (DREYER et al, 2003).

Após o término do procedimento, a mesma sonda pode ser utilizada para a aspiração nasal e, em seguida, oral. Naqueles pacientes que estão recebendo nutrição enteral, interromper a sua administração durante o procedimento, para prevenir vômitos e aspiração pulmonar (OLIVEIRA et al, 2001; DREYER et al, 2003).

Para fluidificar, mobilizar as secreções e estimular a tosse deve ser instilada intrabronquicamente pequenas quantidades de soro fisiológico (até $5 \mathrm{ml})$. Quando há risco de formação de atelectasia, devido às rolhas de secreção, associa-se a utilização do ambu, sempre seguindo os cuidados de assepsia indicados (COSTA, 1999).

Se o tampão persistir, instilar solução bicarbonatada $(0,5 \mathrm{ml}$ de bicarbonato de sódio mais 2,5 de água destilada), seguida de ventilação com ambu (OLIVEIRA et al, 2001).

Entretanto autores como PASSOS et al (2000), DREYER et al (2003) e GONZÁLEZ et al (2004) afirmam que a adequada umidificação dos gases inspirados e a hidratação do paciente evitam a formação de rolhas de secreção. Não é recomendada 
a instilação rotineira de soro fisiológico a $0,9 \%$ na traquéia para fluidificar as secreções, exceto após avaliação da real necessidade do paciente. Esse procedimento provoca hipoxemia, além de infecções, podendo prejudicar o bem estar psicológico do paciente.

É importante ressaltar a existência de sistemas fechados de aspiração que, ligados diretamente ao circuito respiratório, permitem realizar este procedimento sem desconectar o paciente do ventilador mecânico, mantendo a Pressão Expiratória Positiva (PEEP) e diminuindo, assim, o risco de hipoxemia. No entanto, alguns estudos mostraram que sua utilização não influencia a incidência de infecções. Após o término da aspiração, retirar luvas, descartá-las e lavar as mãos em seguida (DREYER et al, 2003).

Reconhecendo a importância deste procedimento e a utilização de diretrizes que venham a minimizar os riscos para o agravo à saúde destes pacientes, consideramos uma questão fundamental para ser investigada.

Objetivamos nesse estudo caracterizar os profissionais que realizam o procedimento de aspiração das VAS nas unidades de urgência e UTI de um Hospital Público da grande Natal/RN e identificar os cuidados executados durante esse procedimento nos setores em estudo.

\section{MÉTODOS}

A pesquisa é do tipo exploratório descritivo com dados prospectivos e abordagem quantitativa, realizada nas Unidades de Urgência e Terapia Intensiva de um Hospital da grande Natal-RN.
A população estudada constou de 14 enfermeiros, 22 técnicos de enfermagem, 14 auxiliares de enfermagem, 03 médicos e 07 fisioterapeutas que realizaram o procedimento de aspiração das VAS no período em estudo.

Para elaborarmos o instrumento ou protocolo de cuidados de aspiração das VAS, tomamos como base os fundamentos preconizados por vários autores, sendo o Guidelines for Prevention of Nosocomial Pneumonia (CDC, 1997) o principal.

A coleta de dados foi realizada nos meses de janeiro e fevereiro de 2005, durante os três turnos, em pacientes com uso de ventilação mecânica. Para tanto, seguimos os princípios éticos e legais que regem a pesquisa em seres humanos, preconizados na Resolução do Conselho Nacional de Saúde $N^{\circ}$. 196/96, manifestada pela aprovação do protocolo registro do Comitê de Ética e Pesquisa (CEP) - UFRN 101-04 (BRASIL, 2000).

Por ser uma pesquisa na qual os dados deveriam ser coletados nas 24 horas, ininterruptamente, necessitamos de uma equipe composta de 07 alunas de iniciação científica do curso de graduação em Enfermagem que se revezaram nos três turnos de trabalho. Antes de começarmos a coleta dos dados, fizemos um treinamento com a finalidade de orientar a equipe, de maneira que todos falassem a mesma linguagem, evitando os vieses na pesquisa. A técnica de coleta de dados utilizada foi a observação estruturada não participante. Para completar os dados durante as observações, utilizamos também um diário de campo.

\section{APRESENTAÇÃO E DISCUSSÃO DOS RESULTADOS}

Quadro 1 - Distribuição das aspirações das vias realizadas durante o estudo. PSCS - Natal/RN, 2005.

\begin{tabular}{|c|c|c|c|c|c|c|}
\hline \multirow{2}{*}{ PROCEDIMENTO (ASPIRAÇÃO) } & \multicolumn{2}{|c|}{ SIM } & \multicolumn{2}{|c|}{ NÃO } & \multicolumn{2}{|c|}{ TOTAL } \\
\hline & $\mathbf{N}$ & $\%$ & $\mathbf{N}$ & $\%$ & $\mathbf{N}$ & $\%$ \\
\hline Lava as mãos antes do procedimento & 65 & 19,46 & 269 & 80,24 & 334 & 100,00 \\
\hline Explica o procedimento ao paciente & 24 & 07,19 & 310 & 92,81 & 334 & 100,00 \\
\hline Interrompe a dieta enteral & 35 & 19,88 & 141 & 80,12 & $176^{*}$ & 100,00 \\
\hline Usa EPIs & 25 & 07,28 & 309 & 92,52 & 334 & 100,00 \\
\hline Dobra a $\mathrm{FiO}_{2}$ ou regula a $100 \%$ & 38 & 11,38 & 296 & 88,62 & 334 & 100,00 \\
\hline Usa luvas estéreis & 310 & 92,81 & 24 & 07,19 & 334 & 100,00 \\
\hline Usa cateter em calibre adequado & 321 & 96,11 & 13 & 03,89 & 334 & 100,00 \\
\hline Usa cateter de aspiração estéril & 325 & 97,30 & 09 & 02,70 & 334 & 100,00 \\
\hline Segue a seqüência tubo, nariz e boca & 281 & 84,13 & 53 & 15,87 & 334 & 100,00 \\
\hline $\begin{array}{l}\text { Usa gaze estéril para limpar secreções do } \\
\text { cateter }\end{array}$ & 196 & 80,83 & 32 & 19,17 & $228^{\star *}$ & 100,00 \\
\hline $\begin{array}{l}\text { Conecta o VM no paciente nos intervalos de } \\
\text { aspiração }\end{array}$ & 270 & 80,84 & 64 & 19,16 & 334 & 100,00 \\
\hline O tempo de aspiração é no máximo 15" & 122 & 36,53 & 212 & 63,47 & 334 & 100,00 \\
\hline Utiliza SF para fluidificar as secreções & 239 & 86,59 & 37 & 13,41 & $276^{*}$ & 100,00 \\
\hline Utiliza ambu estéril & 72 & 72,00 & 28 & 28,00 & $100^{* * x+}$ & 100,00 \\
\hline Descarta o cateter após seqüência de aspiração & 305 & 91,31 & 29 & 08,69 & 334 & 100,00 \\
\hline $\begin{array}{l}\text { Lava o látex com ABD ou SF após o final da } \\
\text { aspiração }\end{array}$ & 245 & 73 & 89 & 5 & 334 & 100, \\
\hline Protege látex após aspiração com embalagem & 278 & 83,23 & 56 & 16,77 & 334 & 100,00 \\
\hline
\end{tabular}


FARIAS, Glaucea Maciel de; FREIRE, Izaura Luzia Silvério; RAMOS; Cristiane da Silva. ASPIRAÇÃO ENDOTRAQUEAL: ESTUDO EM PACIENTES DE UMA UNIDADE DE URGÊNCIA E TERAPIA INTENSIVA DE UM HOSPITAL DA REGIAO METROPOLITANA DE NATAL RN. Revista Eletrônica de Enfermagem, v. 08, n. 01, p. 63 - 69, 2006. Disponível em http://www.fen.ufg.br/revista/revista8 1/original 08.htm

\begin{tabular}{|l|c|c|c|c|c|c|}
\hline limpa e seca & & & & & & \\
\hline Retorna a FiO ${ }_{2}$ inicial & 32 & 84,21 & 06 & 15,79 & 38 & 100,00 \\
\hline Lava as mãos após o procedimento & 168 & 50,29 & 166 & 49,71 & 334 & 100,00 \\
\hline $\begin{array}{l}\text { Anota o procedimento no prontuário, colocando } \\
\text { características das secreções. }\end{array}$ & 92 & 27,54 & 242 & 72,46 & 334 & 100,00 \\
\hline
\end{tabular}

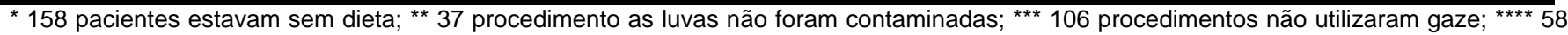
procedimentos não utilizaram SF; ${ }^{\star \star \star \star \star} 296$ não elevaram a FiO2; ${ }^{\star \star \star \star \star 234 ~ p r o c e d i m e n t o s ~ n a ̃ o ~ u t i l i z a r a m ~ a m b u . ~}$

Foram pesquisados 20 passos na realização do procedimento da aspiração das VAS, sendo observados 334 procedimentos.

Como pode ser visto no Quadro 1, das 334 aspirações realizadas, em $269 \quad(80,24 \%)$ os profissionais não lavaram as mãos antes do procedimento.

Esses resultados contradizem o que é preconizado pela literatura, quando afirma que as mãos devem ser lavadas sempre antes e após qualquer procedimento, independente do uso de luvas, pois estas são o principal meio de transmissão das infecções hospitalares (CDC, 1997; ANVISA, 2000, BLOOM \& LIMA, 2003).

Em estudo realizado com enfermeiras sobre a técnica de aspiração, foram encontrados dados semelhantes, quando observaram que somente $56 \%$ delas lavavam as mãos antes do procedimento. As autoras atribuem o fato a algumas crerem que ao usar luvas já estão utilizando uma barreira contra a infecção, o que é um conceito errôneo, já que está demonstrado que o uso de luvas não substitui a lavagem das mãos (GONZÁLEZ et al, 2004).

Em relação a esses passos, chamamos a atenção àqueles que dizem respeito a informar ao paciente previamente à aspiração, percebemos que dos 334 procedimentos em 310 vezes $(92,81 \%)$ essa ação não foi realizada.

Complementando as considerações acerca desse dado, acrescentamos que no estudo de GONZÀLEZ et al (2004) apesar de haver uma concordância em $100 \%$ sobre o fato das enfermeiras na teoria acharem que devem informar ao paciente previamente sobre o procedimento, um pequeno número não o fez na prática. Esse dado é atribuído ao fato de que a alteração do nível de consciência e o menor grau de comunicação das enfermeiras ao paciente com nível de consciência alterado as levam a tomar esse tipo de atitude (GONZÁLEZ et al, 2004, OLIVEIRA et al, 2005).

No que se refere à interrupção da dieta enteral antes da aspiração, das 176 vezes que estava sendo administrada dieta durante o procedimento, em 141 vezes $(80,12 \%)$ esta não foi interrompida.

Sobre esse aspecto, vários autores advertem que a nutrição enteral deve ser interrompida durante o procedimento de aspiração, para prevenir vômitos e aspiração bronco pulmonar (CDC,1997; OLIVEIRA et al, 2001; COLOMBRINI et al, 2003).

Quanto ao uso de EPIs, das 334 vezes que foi realizado o procedimento de aspiração, em 309 $(92,52 \%)$ os profissionais não usaram essa medida de profilaxia, isto é, não utilizaram todos os equipamentos durante a aspiração, que compreendem luvas, gorros, óculos, máscaras e capotes.

Em relação ao uso dos óculos, o Ministério da Saúde diz que devem ser usados durante os procedimentos passíveis de respingo de sangue ou de fluidos corpóreos nas mucosas oral, nasal e ocular do profissional (BRASIL, 1999).

Sobre esse item, GONZÀLEZ et al (2004) obtiveram dados semelhantes, quando avaliaram a competência prática de enfermeiros sobre aspiração. Identificaram nesse estudo que as enfermeiras na teoria consideravam o uso dos óculos necessário, porém, na prática, não os utilizaram.

Quanto ao uso do capote, ele é utilizado, porém os profissionais vestem no início do plantão, só retirando no final. O mesmo capote é utilizado em todos os procedimentos realizados e em diferentes pacientes. Essa prática contradiz as recomendações de pesquisadores (GOMES, 2003; PEREIRA et al, 1999), os quais afirmam que este deve ser imediatamente retirado após o uso e que caso necessite fazer procedimentos em pacientes diferentes deve substituí-lo para evitar infecções cruzadas.

Em relação a $\mathrm{FiO}_{2}$ ser dobrada ou elevada a $100 \%$ antes da aspiração, esse procedimento não foi realizado em 296 vezes $(88,62 \%)$. Sobre essa técnica DREYER et al (2003) salientam que devemos hiperoxigenar o paciente para prevenir hipoxemia e não agravar o seu quadro clínico.

Em relação ao uso de luvas estéreis, a grande maioria, 310 vezes $(92,11 \%)$, fez uso desse passo. Ressaltamos que mesmo sendo essa técnica uma medida profilática no controle das infecções, foram usadas luvas de procedimento não estéril por 12 vezes $(3,83 \%)$.

Nesse estudo, observamos que o cateter utilizado na instituição não era próprio para aspiração das VAS, sendo usada sonda uretral. Quanto ao calibre, estava adequado em $321(96,11 \%)$ vezes. No entanto, em $13(3,89 \%)$ esse cateter foi substituído por sonda nasogástrica ou mesmo sonda uretral de calibre maior ou menor do que o recomendado para o paciente.

Ressaltamos que a literatura consultada recomenda que o cateter ou sonda deva ser macio, de material transparente, com ponta arredondada e múltiplos orifícios em contra-abertura na extremidade distal, que possua válvula digital para controle de pressão do vácuo e que o diâmetro externo não 
exceda $1 / 3$ do diâmetro interno do tubo endotraqueal ou traqueóstomo (SAMPAIO \& FARIA, 1998; COSTA, 1999; THE ROYAL..., 1999; PASSOS et al, 2000; THOMPSON, 2000; GONZÁLEZ et al, 2004).

O cateter de aspiração estéril foi utilizado em 325 vezes $(97,30)$. O cateter de aspiração traqueal pode introduzir microorganismos no trato respiratório baixo. Portanto, deve ser estéril e de uso único (OLIVEIRA et al, 2000).

No que diz respeito seguir a seqüência da aspiração (tubo, nariz e boca), em 281 vezes $(84,13 \%)$ os profissionais seguiram os passos preconizados por alguns autores (OLIVEIRA et al, 2001). A ANVISA (2000) recomenda que quando houver necessidade de aspirar a boca do paciente intubado, esse procedimento deve ser realizado usando uma nova sonda após aspiração traqueal.

Das 228 vezes que foi utilizada gaze para limpar as secreções do cateter durante a aspiração, em 196 (80,83\%) estava estéril. É importante ressaltar que alguns profissionais usaram os invólucros das luvas para limpar as secreções.

Em relação à observação sobre se o ventilador era conectado ao paciente nos intervalos de aspiração, vimos que em 270 (80,84\%) vezes esse procedimento foi realizado de forma correta, isto é, o paciente era ventilado em intervalos para então introduzir novamente o cateter e aspirar outra vez, evitando, assim, a hipóxia.

Por outro lado, observamos que em relação ao tempo de aspiração em $212(63,47 \%)$ vezes os profissionais excederam os 15 segundos de permanência da sonda na traquéia, preconizados pela literatura (SAMPAIO \& FARIA, 1998; COSTA, 1999; THE ROYAL..., 1999; PASSOS et al, 2000; THOMPSON, 2000; GONZÁLEZ et al, 2004). Alguns autores, como SMELTZER \& BARE (2002) e DREYER et al (2003) recomendam um tempo máximo de 10 segundos. Nesse estudo, o tempo mínimo de aspiração das secreções foi de cinco segundo e o máximo de um minuto e trinta segundos.

A bibliografia revisada ressalta amplamente a não instilação de Soro Fisiológico (SF) 0,9\% para fluidificar as secreções. Porém, no nosso estudo, quando buscamos identificar se as secreções eram fluidificadas com SF estéril, das 276 vezes que essa solução foi utilizada em 239 (86,59\%) era estéril.

$\mathrm{Na}$ meta-análise realizada por $\mathrm{OH}$ \& SEO (2003) sobre a aspiração endotraqueal, os autores referem que somente em três dos estudos analisados utilizam o SF.

SOLE et al (2003) em ampla avaliação em 27 hospitais dos Estados Unidos, com 1.665 enfermeiras e fisioterapeutas respiratórios, observaram que $74 \%$ dos centros estudados incluíam nos protocolos a instilação de SF quando as secreções estavam espessas.

THOMPSON (2000) e DREYER \& ZUÑIGA (2003) afirmam que a instilação de solução salina pode ter efeito adverso na Saturação de Oxigênio
$\left(\mathrm{SaO}_{2}\right)$, além do risco de infecção, não devendo ser utilização como rotina ${ }^{3,17}$. Nesse mesmo aspecto, COLOMBRINI et al (2003) ressaltam que a instilação com SF, além de causar hipoxemia e infecções, pode prejudicar o bem-estar psicológico do paciente. Para prevenir a oclusão do tubo traqueal é recomendado fazer a umidificação dos gases inspirados e hidratar o paciente adequadamente.

Das 100 vezes que foi necessária a utilização de ambu estéril ou desinfectado, em 72 (72,00\%) esse artefato estava estéril. FERNANDES et al, (2000) ressaltam que o ambu é usado para ventilar o paciente intubado, em situações de urgência, durante o transporte, para hiperinsuflação nos intervalos das aspirações das VAS e na fisioterapia respiratória; para tanto deverá estar estéril ou desinfectado.

Acrescentando a esses dados, HINRICHSEN (2004) ressalta que as secreções depositadas nesse equipamento podem ser aerolizadas para dentro da árvore traqueobrônquica do paciente ou contaminar as mãos da equipe de atendimento, favorecendo a contaminação cruzada.

Quanto a descartar o cateter após sua utilização, em 305 (91,31\%) ele foi descartado, outras 29 vezes $(8,69 \%)$ deixaram o cateter conectado no látex e pendurado no frasco de aspirador, o que poderia contaminar os outros materiais que estão sendo utilizados ou mesmo o chão; no que se refere a lavar o látex com $A B D$ ou $S F$ no final do procedimento, em 245 vezes $(73,35 \%)$ foi realizado de maneira correta.

Quanto a proteger o látex com embalagem limpa e seca após aspiração, em 278 (83,23\%) esse preceito foi seguido. Essa técnica visa evitar a contaminação do ambiente (DREYER et al, 2003).

Quanto a retornar a FiO2 inicial após a aspiração, das 38 vezes em que foi elevada, em 32 $(84,21 \%)$ vezes esse procedimento foi realizado. A concentração muito elevada de oxigênio pode levar a efeitos tóxicos, como alterações da fisiologia normal, depressão da respiração, diminuição da eritropoese e débito cardíaco, vaso dilatação pulmonar, vaso constrição sistêmica, alteração da formação de surfactante, efeitos cito tóxicos e traqueobronquite (DAVID et al, 2000, SMELTZER \& BARE, 2002).

No que se refere à lavagem das mãos após o procedimento, em 168 vezes $(50,29 \%)$ os profissionais cumpriram esse passo essencial na prevenção de infecções.

Ainda sobre a aspiração das VAS, observamos no prontuário se eram feitas e como eram realizados essas anotações. Em 242 das aspirações (72,46\%) esses dados não foram anotados. OCHOA et al (2003) salientam a importância dos registros de enfermagem no prontuário, informando que os mesmos devem incluir a declaração dos problemas freqüentemente referidos pelos pacientes, os diagnósticos de enfermagem, os tratamentos e as respostas tanto à assistência médica como à de 
enfermagem, expressa o reflexo da avaliação periódica do paciente.

Complementando esses dados, PASSOS et al (2000) reforça a importância de se registrar as características da secreção, descrevendo tipo, cor e odor, podem indicar infecção pulmonar ou fístula traqueoesofágica.

Quando buscamos identificar quais os profissionais que faziam esses procedimentos, vimos que, do total das 334 aspirações realizadas, 162 $(48,50 \%)$ foram realizadas pelos técnicos de enfermagem, 82 (24,55\%) auxiliar de enfermagem, 75 $(22,46 \%)$ fisioterapeuta, 12 (3,59\%) enfermeiro e 03 (0,90\%) pelo médico.

RIBEIRO \& PEDRÃO (2005) ressaltam que o pessoal de nível médio, apesar de ser o menos qualificado, é executor da maior parte das ações na assistência direta aos pacientes internados e que necessitam de cuidados intensivos. Quanto ao enfermeiro, mesmo em unidades que prestam cuidado à pacientes graves, continua delegando tarefas, atuando mais na administração da unidade do que nos cuidados direto ao paciente.

Completando os dados sobre o procedimento da aspiração das secreções das VAS, observamos que não havia um horário pré-determinado e que na maioria das vezes o paciente só foi aspirado quando apresentava secreção visível no tubo. Com exceção do fisioterapeuta, os demais profissionais aspiravam ao paciente sem que tivesse sido feita uma ausculta prévia ou após o procedimento.

Sobre essa temática, COLOMBRINI et al (2003), COSTA (1999), PASSOS et al (2000) e THOMPSON (2000) afirmam que a aspiração expõe o paciente a riscos como hipóxia, lesões da mucosa traqueal, atelectasia e infecções e só deve ser realizada quando necessário. ZEITOUN et al (2001) ressaltam que a necessidade de aspiração é determinada principalmente pela observação visual do acúmulo de secreções e pela ausculta pulmonar, para determinar a presença de secreções ou obstruções das vias aéreas. Alguns autores estipulam uma freqüência de 4/4 horas e quando necessário para que o paciente seja aspirado (OLIVEIRA et al, 2001). Outros justificam a necessidade de estipular um tempo porque geralmente esse procedimento é realizado por técnicos ou auxiliares de enfermagem, a priori por não terem o preparo para fazer um julgamento clínico através da ausculta pulmonar, passa a realizá-la somente quando necessário (WILLEMEN \& CRUZ, 2004).

\section{CONCLUSÕES}

Observamos 334 aspirações das VAS, das quais em $269(80,24 \%)$ os profissionais não realizaram lavagem prévia das mãos; os procedimentos não foi informado previamente em 310 $(92,81)$ pacientes; em 176 dietas administradas no momento da aspiração, em 141 vezes 80,12\%) não foram interrompidas durante a aspiração; 309 vezes $(92,52 \%)$ os profissionais não utilizaram todos os EPIs; em $296(88,62 \%)$ a $\mathrm{FiO}_{2}$ não foi dobrada ou elevada a 100\%; em 310 vezes $(92,81 \%)$ os profissionais utilizaram luvas estéreis; 325 (97,30\%) fomos usados cateter de aspiração estéril; 281 $(84,13 \%)$ fomos seguidas a seqüência de aspiração (tubo, nariz e boca); das 228 vezes que foi utilizado gaze para limpar as secreções, em 196 (80,83\%) esse material estava estéril; o ventilador mecânico foi conectado no paciente nos intervalos de aspiração em 270 (80,84\%); o tempo de aspiração excedeu 15 segundos em $212(63,47 \%)$ das vezes; mesmo sendo incorreto o uso do SF, este foi utilizado por 276 vezes, em 239 (88,89\%) estava estéril; das 100 vezes que foi necessário à utilização de ambu, em 72 (72,00\%) estava estéril; o cateter foi descartado após sua utilização, em 305 (91,31\%); a lavagem do látex com ABD e SF no final da aspiração ocorreu em 245 vezes (73,35\%); 278 vezes $(83,23 \%)$ o látex foi protegido com embalagem limpa e seca; das 38 vezes que a $\mathrm{FiO}_{2}$ foi elevada, foi retornada em 32 $(84,21 \%)$ das vezes; as mãos foram lavadas, após o procedimento em 168 vezes (50,29\%). 162 procedimentos $(48,50 \%)$ foram realizados pelos técnicos de enfermagem; 82 (24,55\%) auxiliar de enfermagem, 75 (22,46\%) fisioterapeuta, 12 (3,59\%) enfermeiro e $03(0,90 \%)$ pelo médico.

Inferimos, pois, que o procedimento de aspiração realizada pelos profissionais de saúde nas unidades estudadas, isto é, na UTI e setor de urgência, não utilizam os passos adequadamente como preconiza a ANVISA (2000), entre outros autores consultados, no momento da aspiração das secreções endotraqueal, especialmente no que se refere à lavagem das mãos. Sendo assim, os pacientes submetidos a esse procedimento correm um sério risco de adquirirem infecção nosocomial.

\section{REFERÊNCIAS BIBLIOGRÀFICAS}

ANVISA. Agência Nacional de Vigilância Sanitária. Curso básico de controle de infecção hospitalar. Caderno B: principais síndromes infecciosas hospitalares. Brasília, 2000, p. 31-54. Disponível em: $<$ http://www.anvisa.gov.br/> [Acesso em 18 mai. 2004].

BLOM, B.C.; LIMA, S.L. de. Lavagem das mãos. In: COUTO, R.C.; PEDROSA, T.M.G.; NOGUEIRA, J.M. Infecção hospitalar e outras complicações nãoinfecciosas da doença: epidemiologia, controle e tratamento. $3^{a}$ ed. Rio de Janeiro: Medsi, 2003. p. 481-496.

BRASIL. Ministério da Saúde. Coordenação Nacional de DST e AIDS. Manual de condutas: exposição ocupacional a material biológico: hepatite e HIV. Brasília; 1999.

Ministério da Saúde. Comissão Nacional de Ética em Pesquisa. Res. CNS 196/96. Brasil, Brasília, 2000. (Série cadernos técnicos). 
CDC. Centers for Diseases Control and Prevention. Guidelines for prevention of nosocomial pneumonia. v. 46, 1997 .

COLOMBRINI, M.R.C. et al. Assistência de enfermagem a pacientes em ventilação mecânica. In: ZUÑIGA, Q.G.P. Ventilação mecânica básica para enfermagem: São Paulo: Atheneu, 2003, p.51-56.

COSTA, D. Fisioterapia respiratória básica: São Paulo: Atheneu, 1999.

DAVID, C.M.N. et al (Org.). Complicações da ventilação mecânica. II Consenso Brasileiro de Ventilação Mecânica. Jornal de pneumologia. São Paulo, v. 26, supl. 2, p. 45-62, 2000.

DREYER, E.; ZUÑIGA, Q.G.P. Ventilação mecânica. In: CINTRA, E. de A.; NISHIDE, V.M.; NUNES, W.A. Assistência de enfermagem ao paciente gravemente enfermo. 2 ed. São Paulo: Atheneu, 2003, p. 351-366. DREYER, E. et al. Técnicas de enfermagem na ventilação mecânica. In: ZUÑIGA, Q.G.P. Ventilação mecânica_para enfermagem. São Paulo: Atheneu, 2003, p. 41-49.

FERNANDES, A.T.; ZAMORANO, P.O.; TOREZAN FILHO, M.A. Pneumonia hospitalar. In: FERNANDES, A.T. (Org.). Infecção hospitalar e suas interfaces na área da saúde. São Paulo: Atheneu, 2000, p.516-555. GOMES, D.L. de C. Precauções e isolamento de pacientes. In: Infecção hospitalar e outras complicações não-infecciosas as doença: epidemiologia, controle e tratamento. Rio de Janeiro: Medsi, 2003, p. 469-479.

GONZÁLEZ, N.A. et al. Avaliação da competência prática e dos conhecimentos científicos das enfermeiras de uma $\mathrm{UCl}$ sobre a aspiração endotraqueal. Cuidado Intensivo, São Paulo, v. 1, n .4, p .7-17, 2004.

HINRICHSEN, S.L. Aspectos éticos e jurídicos dos processos infecciosos hospitalares e suas relações com a vigilância e a qualidade assistencial. In: HINRICHSEN, S.L. Biossegurança e controle de infecções: risco sanitário hospitalar. Rio de Janeiro: Medsi, 2004, p. 273-281.

OCHOA VIGO, K.; PACE, A.E.; SANTOS, C.B. dos. Análise retrospectiva dos registros de enfermagem em uma unidade especializada. Revista Latino Americana de Enfermagem, v. 11, n. 2, p. 184-191, 2003.

$\mathrm{OH}$, H.S.; SEO, W.S. A meta-analysis of the effects of various interventions in preventing endotracheal suction-induced hypoxemia. Journal of Clinical Nursing. v. 12, n. 6, p. 912-24, 2003.

OLIVEIRA, A.C. de; ARMOND, G.A.; TEDESCO, L.A. Procedimentos nas vias respiratórias. In: MARTINS, M.A. Manual de infecção hospitalar: epidemiologia, prevenção e controle. 2 ed. Rio de Janeiro: Medsi, 2001, p. 343-353.

OLIVEIRA, P.S.; NÓBREGA, M.M.L.; SILVA, A.T.; FERREIRA FILHA, M. O. Comunicação terapêutica em enfermagem revelada nos depoimentos de pacientes internados em centro de terapia intensiva. Revista Eletrônica de Enfermagem, v. 07, n. 01, p.
54-63,

2005.

Disponível

em

http://www.fen.ufg.br/revista/revista7 1/v7n1.htm

[Acesso em: 18 mai. de 2005].

PASSOS, E. et al. Papel da enfermagem na assistência ao paciente em ventilação mecânica. II Consenso Brasileiro de Ventilação Mecânica. Jornal de Pneumologia. São Paulo, v. 26, sup. 2, p. 27-34, 2000.

PEREIRA, T.M. et al. Avaliação da adoção das medidas de precauções padrão em categorias específicas de profissionais de saúde. Revista Eletrônica de Enfermagem (on line), v.1, n.1, 1999. Disponível

http://www.fen.ufg.br/revista/revista1 1/05.htm

[Acesso em 15 ago. 2004].

RIBEIRO, M.I.L.C.; PEDRÃO, J.L. O ensino de enfermagem no Brasil: enfoque na formação de nível médio. Revista Nursing, v. 82, n. 8, p. 125-28, 2005.

SAMPAIO, L.A.B.N; FARIA, M. de F.G. de. Atuação da enfermagem em ventilação mecânica. In: AMARAL, R.V.G.; AULER JÚNIOR, J.O.C. Assistência ventilatória mecânica. Rio de Janeiro: Atheneu, 1998, p. 339-353.

SMELTZER, S.C.; BARE, B.G. Brunner e Suddarth: tratado de enfermagem médico-cirúrgica. 9 ed. Rio de Janeiro: Guanabara Koogan, 2002.

SOLE, M.L. et al. A multisite survey of suctioning tecniques and airway management practices. American Journal of Critical Care. v. 12, n.3, p. 220230, 2003.

THE ROYAL FREE HAMPSTEAD NHS TRUST. Guidelines for tracheal suction. 1999. Disponível em: http://www.bahnon.org.uk/Professional\%20Guidelines ITrachealSuction.doc [Acesso em: 09 jan. de 2005].

THOMPSON, L. Suctioning adults with an artificial airway. The Joanna Briggs Institute for Evidence Based Nursing and Midwifery. Best Practice. v. 4, n. 4, 2000, 6p. Disponível em: http://www.joannabriggs.edu.au [Acesso em: 09 jan. de 2005].

WILLEMEN, R.L.A.; CRUZ, I.C.. Produção científica de enfermagem sobre aspiração de vias aéreas: implicações para a (o) enfermeira (o) de cuidados intensivos. 2001. Monografia (Especialização) Escola de Enfermagem, UFF, 2001. Disponível em: $<$ http://www.uff.br/nepae/bnn04esp02.htm> [Acesso em: 15 ago. de 2004].

ZEITOUN, S.S. et al. Incidência de pneumonia associada à ventilação mecânica em pacientes submetidos à aspiração endotraqueal pelos sistemas aberto e fechado: estudo prospectivo - dados preliminares. Revista latino-americana de Enfermagem. v. 9, n. 1, p. 46-52, 2001.

Artigo original recebido em 10/04/2006 Aprovado para publicação em30/04/2006 